\title{
Significantly improved estimates of neutron capture cross sections relevant to the $\boldsymbol{r}$ process
}

\author{
A. Couture $\odot,{ }^{1}$ R. F. Casten $\odot, 2,3$ and R. B. Cakirli ${ }^{4}$ \\ ${ }^{1}$ Los Alamos National Laboratory, Los Alamos, New Mexico 87545, USA \\ ${ }^{2}$ Wright Lab, Yale University, New Haven, Connecticut 06520, USA \\ ${ }^{3}$ Facility for Rare Isotope Beams, Michigan State University (MSU-FRIB), East Lansing, Michigan 48823, USA \\ ${ }^{4}$ Department of Physics, Istanbul University, Istanbul 34134, Turkey
}

(Received 6 September 2021; accepted 27 October 2021; published 18 November 2021)

\begin{abstract}
Background: The $r$ process is thought to be a dominant mechanism for the production of medium mass and heavy nuclei. Despite extensive study, neither the process nor its site, and, consequently, the origin of these elements, is well understood. One of the principal reasons is the lack of adequate knowledge of neutron capture cross sections in neutron-rich nuclei, especially up to a few hundred keV. Existing statistical model calculations differ widely and are unreliable more than a few nucleons beyond stability.

Purpose: To provide a new, more reliable empirical method to estimate neutron capture cross sections.

Method: To use an entirely empirical approach by exploiting a newly discovered correlation between twoneutron separation energies and neutron capture cross sections.

Results: It is shown that there is a compact correlation between neutron capture cross sections and $S_{2 n}$ for $S_{2 n}$ values from 10 to $16 \mathrm{MeV}$, encompassing nuclei in five mass regions comprising seven classes of nuclei from $A \approx 110$ through the actinides over Maxwellian energy distributions from $k T=5$ to $100 \mathrm{keV}$. As a consequence, many unknown cross sections can be predicted by interpolation, with accuracies on the order of $\pm 25 \%$. In other cases, the cross sections can still be predicted with greatly improved accuracy compared to current models. Finally, it is shown that, in very neutron-rich nuclei, measurements of $S_{2 n}$ can replace more difficult or impossible neutron capture measurements to estimate $r$-process abundances.

Conclusions: A new approach to estimate neutron capture cross section allows much improved predictions of these key ingredients into $r$-process calculations, perhaps providing an enhanced ability to model this process and to better define its site(s).
\end{abstract}

DOI: 10.1103/PhysRevC.104.054608

\section{INTRODUCTION}

Reactions on atomic nuclei play a key role in understanding environments as diverse as hydrostatic stellar interiors, nuclear reactors, and explosions that occur in the death throes of a massive star. In astrophysics, numerous reactions are critical to answering basic questions concerning stellar environments and the origin of the elements. These questions have engaged nuclear astrophysics since Eddington [1] first suggested that nuclear reactions might be the key mechanism to explain stellar lifetimes and luminosity, and decades later, the modern theory of nucleosynthesis was independently developed by Cameron [2] and Burbidge et al. [3].

While major strides have been made since these seminal works, significant uncertainties and open questions remain, particularly for elements heavier than iron. Since fusion reactions above iron are endothermic, they cannot prevent gravitationally induced stellar collapse. Moreover, even if

Published by the American Physical Society under the terms of the Creative Commons Attribution 4.0 International license. Further distribution of this work must maintain attribution to the author(s) and the published article's title, journal citation, and DOI. fusion could contribute energetically, the increased Coulomb barriers with increasing $Z$ of stellar constituents would require much larger energies to allow fusion reactions. The primary mechanism to produce the heavier elements is therefore (exothermic) neutron capture. No Coulomb barrier hindrances are involved and capture reactions can occur at the low kinetic energies typical of stellar interiors. Therefore, critical to understanding the origin of most of the elements beyond iron, and hence the stellar environments in which they are synthesized, is the quantitative understanding of neutron capture cross sections in the energy range (typically $\mathrm{keV}$ to a few $\mathrm{MeV}$ ) relevant to their stellar sites.

Two broad classes of neutron capture reactions are slow neutron capture (the $s$ process) and rapid neutron capture (the $r$ process). The time scales involved are relative to $\beta$ decay which, when it occurs, arrests subsequent capture on a given element and shifts the nucleosynthetic process to the next higher $Z$ element. The $s$ process centers on stable and near-stable nuclei and competes with $\beta$ decay, while the $r$ process outruns $\beta$ decay towards neutron-rich nuclei until special conditions apply. Importantly, therefore, the $r$ process extends to isotopic regions where $(n, \gamma)-(\gamma, n)$ equilibrium occurs in hot environments (e.g., core-collapse supernovae) or to the neutron dripline in cold scenarios, such as neutron star mergers. 
Recent rediscovery of the so-called intermediate or $i$ process has made this even more challenging [4,5]. This is a neutron capture process predicted during explosive shell burning which, like the $s$ process, operates in $\beta$-decay equilibrium, but at orders of magnitude higher neutron density, such that the reactions of interest often take place 5+ neutrons away from stability. For both the $i$ and the $r$ processes, not all nuclides are equally important to final abundances but many contribute, and recent studies have attempted to systematically highlight the specific cross sections to which the nucleosynthetic trajectory is particularly sensitive (see, e.g., Ref. [6]).

The importance of accurate neutron capture cross sections for interpreting $r$-process abundances and, hopefully, determining the $r$-process site or sites has been clearly demonstrated. While earlier work argued that neutron capture rates were largely secondary to neutron separation energies, as the burning would take place in nuclear statistical equilibrium (NSE) (see, e.g., [7]), more recent efforts have shown that, even if NSE is reached in an $r$-process event, during fall-out from NSE, the neutron capture cross sections once again become important [8]. Surman et al. demonstrated that uncertainty in neutron capture rates on a multitude of rare-earth isotopes could each have appreciable impact on the predicted final abundances, even in a so-called "hot" $r$ process where NSE is achieved [9]. Further studies by Mumpower et al. demonstrated that individual neutron capture rates continue to impact final predicted abundances in a "cold" $r$ process where NSE is not achieved [10]. This type of environment may be more typical in a scenario where a neutron-star merger generates an $r$ process. Liddick et al. demonstrated that uncertainties of a factor of 10 or greater in neutron capture cross sections are sufficient to obscure salient details in the predicted rare earth abundances [11]. The data they presented show clear improvements in constraints that can be achieved if the neutron capture cross section uncertainties can be reduced to a factor of 2 from a factor of 10 (for details, see Figure 4 in [11] and the surrounding discussion).

In addition to the importance of neutron capture for element synthesis, terrestrial applications also require reliable neutron capture data on both stable and unstable nuclei. As reactor design has advanced and evolved beyond relatively simple, light-water, thermal reactors with low burn-up, the number of nuclei that participate in both the energy production and the safety envelope have increased, requiring reaction cross sections on a broader range of shorter-lived nuclei. Sensitivity studies have identified needs in the actinides $[12,13]$. Finally, national security applications require neutron capture cross sections, both for stockpile stewardship and nuclear forensics [14]. Reliable prediction of cross sections for isotopes just off stability continues to be of pressing interest.

Unfortunately, one faces a dual challenge here. First, while capture measurements on stable targets are usually straightforward, those on unstable targets are often either extremely difficult, very time consuming, or impossible with foreseeable technology. The relevant incident neutron energy is quite low, nominally a few to hundreds of $\mathrm{keV}$, requiring detailed resonance spectroscopy of neutron states. Time-of-flight measurements are typically the gold-standard for capture cross section measurements due to their differential information, but even for $s$-process studies, orders of magnitude higher neutron densities would be required to complete measurements on all of the necessary branch points [15]. Activation measurements, particularly with accelerator mass spectroscopy for counting, can offer increased sensitivity, but the techniques are limited to those cases with favorable decay branchings and lifetimes [16-18]. Finally, while initial concepts have been floated for how to perform a direct neutron capture experiment with a radioactive ion beam, none are currently under construction [19].

On the theoretical side, prediction of $(n, \gamma)$ cross sections is daunting since capture produces final nuclei at compound nuclear excitation energies near or above the neutron separation energy, that is, in the multi-MeV range, where the level density is extraordinarily high, the structure of individual levels is largely unknown, and computational challenges of existing nuclear structure models abound.

One thus resorts to statistical nuclear models and computational approaches such as Hauser-Feshbach (H-F) [20,21]. These calculations require a knowledge or estimate of statistical approximations of the structural features including spin-dependent level densities, optical model parameters, and $\gamma$-ray de-excitation routes and probabilities. In most cases, the models parameterize these quantities. For stable nuclei, where cross sections are known experimentally, such parametrized calculations, perhaps not surprisingly, generally reproduce the data reasonably well (typically to within 25\%). Beyond stability, there are few experimental calibration points, less control or constraints on the H-F variables, and, consequently, different $\mathrm{H}-\mathrm{F}$ calculations can quickly diverge in their predictions. The problem exacerbates very quickly, leading to rapid disagreements of predictions reaching an order of magnitude within just a few nucleons beyond stability. For decades, extensive work has been done to improve and stabilize the H-F approach, with many added structural degrees of freedom, but adequate convergence is still lacking. Yet, the $r$ process often involves nuclides ten or more neutrons beyond stability where the rapidly growing uncertainty renders it very difficult to distinguish competing models of nucleosynthetic sites and timescales.

We will present below a new approach, introduced in Ref. [22], that obviates these problems for a wide range of nuclei and neutron energies. It can yield much more accurate predictions of neutron capture cross sections, including predictions for many nuclei by a process of interpolation, and hence significantly improve the calculation of $r$-process abundances and their uncertainties. It further suggests a simple way to estimate these cross sections by easy measurements in currently unknown nuclei.

\section{THE NEUTRON CAPTURE CROSS SECTION- $\boldsymbol{S}_{2 n}$ CORRELATION}

As just noted, the present paper originated in a previous study [22] where we introduced an alternate, purely empirical and parameter-free, correlation of observables that seems to bypass the difficulties discussed above and offers a much easier and more reliable way to estimate many of the needed cross sections, even for nuclei well-off stability. Reference 


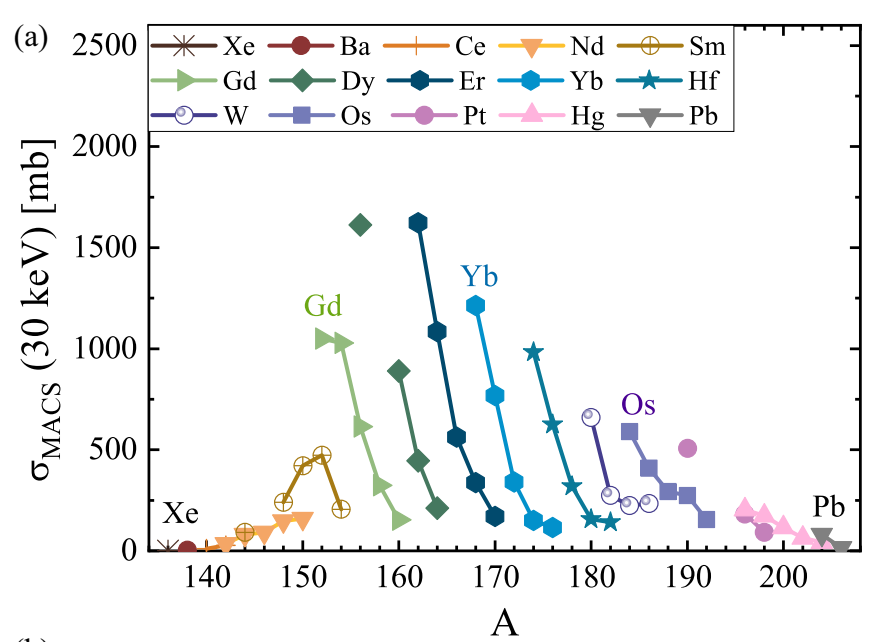

(b)

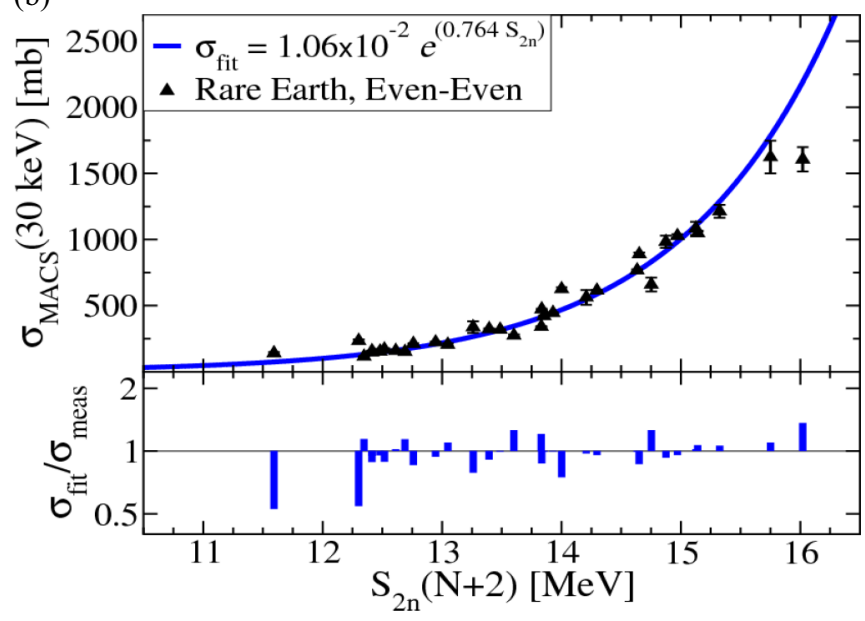

FIG. 1. (a) Experimental Maxwellian-averaged cross section (MACS) for neutron capture evaluated at $k T=30 \mathrm{keV}$ are shown versus atomic mass $A$ for 57 even-even nuclei [23]. (b) The same MACS is shown plotted versus the two-neutron separation energy $\left(S_{2 n}\right)$ for the nucleus with two more neutrons than the capturing nucleus. The nuclei shown here (33) are even-even deformed nuclei between $\mathrm{Nd}$ and $\mathrm{W}$ [23-25]. The figure shows a simple best exponential fit function, discussed in the text in Sec. III B, with the lower panel of (b) indicating the deviation of each point from the fit.

[22] focused primarily on one region of even-even deformed rare earth nuclei at one neutron energy, albeit briefly showing some results for other regions. The present paper builds on this by presenting a coherent analysis that combines all the regions considered in Ref. [22], and others, in a unified framework and for a range of relevant temperatures, covering thermal Maxwell-Boltzmann distributions peaked at neutron energies from 5-100 keV.

To give a concrete context to this discussion, it is useful to look at a given region. Figure 1(a) shows the empirically evaluated Maxwellian-averaged cross section (MACS) [23] (hereafter referred to as KADoNiS, which will be discussed further below) at $k T=30 \mathrm{keV}$ as a function of atomic mass $A$. This is a typical neutron energy for the $s$ process where capture cross sections are usually well-measured. Clearly, while there are some trends in Fig. 1(a), the cross sections range over large factors with little correlation with mass and no clear route to extrapolation.

Figure 1(b) (based on Ref. [22]) shows the new perspective. Instead of plotting the cross sections against a variable that merely identifies the nuclei as in Fig. 1(a), we plot the cross section for each nucleus against another observable pertaining to that nucleus, namely the two-neutron separation energy, $S_{2 n}$.

We note that, for capture on a nucleus with $(Z, N)$, leading to the nucleus $(Z, N+1)$, we use the $S_{2 n}$ value for the separation of two neutrons from the nucleus $(Z, N+2)$. In this way, the separation energy focuses on the average binding energy of the $(N+1)$ th neutron that is actually captured. The choice of $S_{2 n}$ instead of $S_{n}$ was dictated by the desire to avoid issues associated with varying spins and parities of the intermediate odd- $N$ nucleus. The same choice of $(N+2)$ will apply to oddeven nuclei discussed below. For capture on odd $-N$ nuclei, that is, even-odd and odd-odd species, for the same reason, we will use $S_{2 n}(N+1)$.

The figure exhibits a striking correlation between masses and neutron capture cross sections as discussed in Ref. [22]. The figure also shows a function fitted (see below) to these data which describes the empirical trend very well. The compactness of the correlation provides a new method for predicting unmeasured capture cross sections, at a potentially higher level of accuracy than heretofore available, either by interpolation, where the uncertainty is largely determined by the scatter of the points, or by extrapolation where the accuracy is related to the appropriateness of the fitted function. In both contexts we note that separation energies are among the first observables that can be determined for newly studied nuclei and hence are often known for nuclei quite far from stability. Thus the correlation offers the opportunity to make predictions for neutron capture cross sections that are very difficult or impossible to measure.

The primary purpose of the present paper is to significantly extend this correlation to provide a unified set of results spanning a wide region of the medium mass and heavy elements and the important range of neutron energies from 5-100 keV. We describe this process in the next section. In Sec. IV we show the results. In Sec. V we discuss the results, and methods to predict unknown cross sections. We use these to make a large number of predictions for unknown cross sections, particularly by interpolation. Finally, we comment on the physics reasons behind the success of the correlation. We summarize the conclusions in Sec. VI.

\section{METHODS AND RESULTS FOR THE $30 \mathrm{keV}$ DATA}

\section{A. Data sources}

The primary source for the neutron capture cross sections was the Karlsruhe Astrophysical Database of Nucleosynthesis in Stars (KADoNiS) [23]. This database provides recommended, experimentally based, Maxwellian-averaged neutron-capture cross sections over the range of $k T=$ $5-100 \mathrm{keV}$. While the majority of the data in KADoNiS is experimental, in some cases, where measurements are not available, KADoNiS provides "semiempirical estimates" by 
normalizing theoretical calculations to nearby direct measurements. There are also some values provided strictly from theory.

We wanted our approach to be purely data driven, and hence we use only those data that come from direct measurement. We excluded those KADoNiS cross sections that were semiempirical estimates or purely theoretical values. This applies as well to the energy dependence of the cross sections. In some cases, there may be data at a single neutron energy, but the energy dependence in the KADoNiS database may have been derived from scaling theoretical calculations to the measured energy. In these cases, we have only included the measured value at $30 \mathrm{keV}$, but not those lower and higher energy data based on a model energy dependence.

Finally, note that KADoNiS does not provide neutron capture results above $A=210$. For the actinides, we have taken values of the MACS at $30 \mathrm{keV}$ obtained with the NucRates tool using the ENDF/B-VII.1 library [26,27]. It is important to note that ENDF is a full evaluation and incorporates both theory and experimental results, which is a deviation from the approach used in KADoNiS. In this work we only show those isotopes for which direct measurements exist and were included in the actinide values. The manner in which uncertainties are assigned in the ENDF framework does not translate well to this study. Instead, for this work, a coarse uncertainty of 10,15 , or $30 \%$ was assigned to the actinide cross sections based on the number of measurements and the experimental reliability.

Two-neutron separation energy values $\left(S_{2 n}\right)$ were all taken from the 2016 Atomic Mass Evaluation (AME2016) [24,25]. As with KADoNiS, the AME2016 mass tabulations also sometimes use estimated or extrapolated values. We did not use them, relying solely on directly measured and evaluated values. Because the mass uncertainties are small relative to $S_{2 n}$ values, we do not report them and did not include them in our analysis.

All cross sections, uncertainties, and $S_{2 n}$ data used in this work are listed in the Appendix.

\section{B. Exponential fit to the $30 \mathrm{keV}$ rare earth data}

To formalize the quality of the correlation and to facilitate later predictions, the data for the $30 \mathrm{keV}$ MACS for the eveneven rare earth deformed nuclei in Fig. 1(b) were fit to an exponential function of the form

$$
\sigma_{\text {fit }}=p_{0} e^{p_{1} S_{2 n}}
$$

This functional form was chosen because of its close connection to most estimates of the level density and the likely connection between $S_{2 n}$ and level densities (see discussion in Sec. V). The least-squares fit of $p_{0}$ and $p_{1}$ values included weights based on experimental cross section uncertainties. The coefficients $p_{0,1}$ are highly correlated and hence the true uncertainties were obtained by a covariance analysis. Since the system is overconstrained, the fit uncertainties in the predicted cross sections are exceptionally small-typically $1-2 \%$. Of course, the quality of the correlation, in the end, is determined by the empirical scatter of the data. To assess this, we show the residuals from the fit in the bottom panel

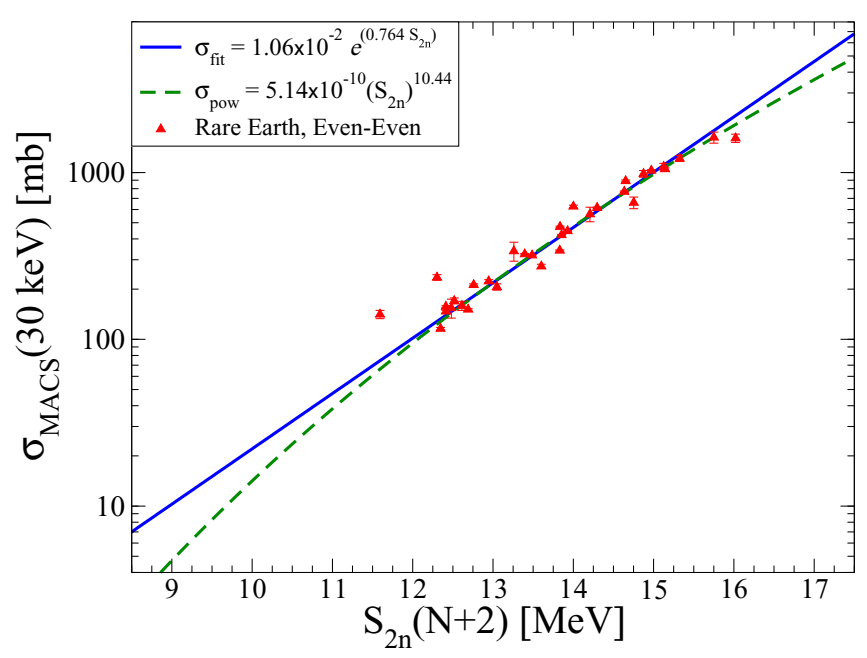

FIG. 2. Comparison of the exponential fit function from Fig. 1(b) (solid/blue) and the power law fit function used in Ref. [22] (dashed/green). The choice between the two for low $S_{2 n}$ values can be decided by a small number of new neutron capture measurements in the region of $S_{2 n}=8-10 \mathrm{MeV}$.

of Fig. 1(b), most of which are within $25 \%$ of the curve. While this is a remarkably small spread, it is much larger than the formal fit function uncertainty; the fit uncertainty does not capture the physics driving the variations. Hence, to reflect the true uncertainty, we use the residuals to assess the uncertainties in predicted cross sections below rather than the formal fit uncertainties.

We note briefly that, in Ref. [22], we used a power law fit function while the present work uses an exponential. The two functions are almost identical over most of the $S_{2 n}$ range in the figures but do deviate slightly at the highest and, especially, the lowest $S_{2 n}$ ranges. For $A>100$, these low $S_{2 n}$ values correspond to weakly bound, neutron-rich nuclei off stability well into the $r$-process region. In Fig. 2, we compare the fit functions. The deviation at, say $9 \mathrm{MeV}$, has grown to approximately a factor of 2 . While this is of concern for accurate cross section estimates, even such an uncertainty is small compared to those from statistical model approaches. As noted, in this paper we use the exponential form. We stress that even a few neutron capture measurements for $S_{2 n}$ values in the region $8-10 \mathrm{MeV}$ would help distinguish between the two (or other) extrapolations.

\section{EXTENDING THE RESULTS-OTHER REGIONS, EXTENDED NEUTRON ENERGY RANGE}

\section{A. Other mass regions-30 $\mathrm{keV}$ data}

In Ref. [22], we noted briefly that there was a tight correlation between $S_{2 n}$ and neutron capture cross sections in four other classes of nuclei (above $A \approx 110$ ) but the correlations differed from those for the rare earth region. Further study that we report here has revealed that there is a closer correspondence between different regions than heretofore recognized. In particular, to reasonable accuracy, the $30 \mathrm{keV}$ data for these four additional regions can be brought into close correspon- 
TABLE I. Table of parameters of the fits. Values fit for a particular class are indicated in bold. The fit uncertainties in $p_{0}$ and $p_{1}$ are 0.0005 and 0.003 , respectively. The reader is reminded that the fit parameters are highly correlated and the fit uncertainties are not representative of the predicted cross section uncertainties. For details, see Sec. III B.

\begin{tabular}{lcccc}
\hline \hline Region & $Z-N$ & $p_{0}(\mathrm{mb})$ & $p_{1}$ & $p_{2}(\mathrm{MeV})$ \\
\hline Rare earth & even-even & $\mathbf{1 . 0 6} \times \mathbf{1 0}^{-\mathbf{2}}$ & $\mathbf{0 . 7 6 4}$ & - \\
& even-odd & $1.06 \times 10^{-2}$ & 0.764 & $+\mathbf{1 . 0 4} \pm<.01$ \\
& odd-even & $1.06 \times 10^{-2}$ & 0.764 & $\mathbf{+ 1 . 7 4} \pm<.01$ \\
Te-Xe-Ba & even-even & $1.06 \times 10^{-2}$ & 0.764 & $\mathbf{- 3 . 2 7} \pm<.01$ \\
Ce-Nd-Hg & even-even & $1.06 \times 10^{-2}$ & 0.764 & $\mathbf{- 1 . 9 2} \pm 0.02$ \\
Pt-Os & even-even & $1.06 \times 10^{-2}$ & 0.764 & $\mathbf{- 0 . 5 6} \pm 0.03$ \\
Actinides & even-even & $1.06 \times 10^{-2}$ & 0.764 & $\mathbf{+ 2 . 9 6} \pm 0.07$ \\
\hline \hline
\end{tabular}

dence to the even-even rare earth data of Fig. 1, by introducing a single constant shift in $S_{2 n}$ for each region. Hence, Eq. (1) was modified to

$$
\sigma_{\text {fit }}=p_{0} e^{p_{1}\left(S_{2 n}+p_{2}\right)}
$$

where $p_{0,1}$ were kept fixed from the original fit of the eveneven rare earth nuclei and only the term $p_{2}$, which shifts the effective $S_{2 n}$, was fit for each region. Moreover, we have now also found that two other important regions can be similarly accommodated. We will discuss the rationale for these shifts below. Here, we treat them as empirical findings.

For each class, the shifts were obtained by a least squares fit to optimize the agreement of the shifted data with the exponential fit function discussed above. Again, each class was an overconstrained system, with shift uncertainties primarily signifying the uncertainties in the cross-section measurements. Typical shift uncertainties were in the range of $20 \mathrm{keV}$ or less, with the actinides being somewhat larger. The impact of these uncertainties on predicted cross sections is again at the $\approx 1 \%$ level, which is substantially less than the scatter in the data.

The five classes of nuclei previously considered are the even-even deformed rare earth nuclei, the even-even $\gamma$-soft $\mathrm{Pt}$ and Os nuclei, the even-even Te-Xe-Ba nuclei that are transitional from spherical to near $\gamma$-soft, the even-even spherical $\mathrm{Ce}, \mathrm{Nd}$, and $\mathrm{Hg}$ nuclei, and the even-odd deformed rare earth nuclei. The new classes comprise the even-even actinide nuclei and the odd-even deformed rare earth nuclei. The shifts for all seven classes are given in Table I. Together these comprise five regions with seven classes of nuclei starting around $A \approx 110$ and ranging to the actinides. The result for a region of odd- $Z$ nuclei is particularly noteworthy for two reasons: it represents the first successful connection between $S_{2 n}$ and the capture cross sections for odd- $Z$ nuclei, which is essential for addressing predictions for nuclear astrophysics, and, by giving the first shift for odd- $Z$ nuclei, it expands the reach of an important extension of these results to nuclei with unknown neutron capture cross sections (see below). The resulting correlation for 97 nuclei covering seven classes listed in Table I is shown in Fig. 3. We stress that the fit function shown in Fig. 3 is the same as that used for the even-even rare nuclei in

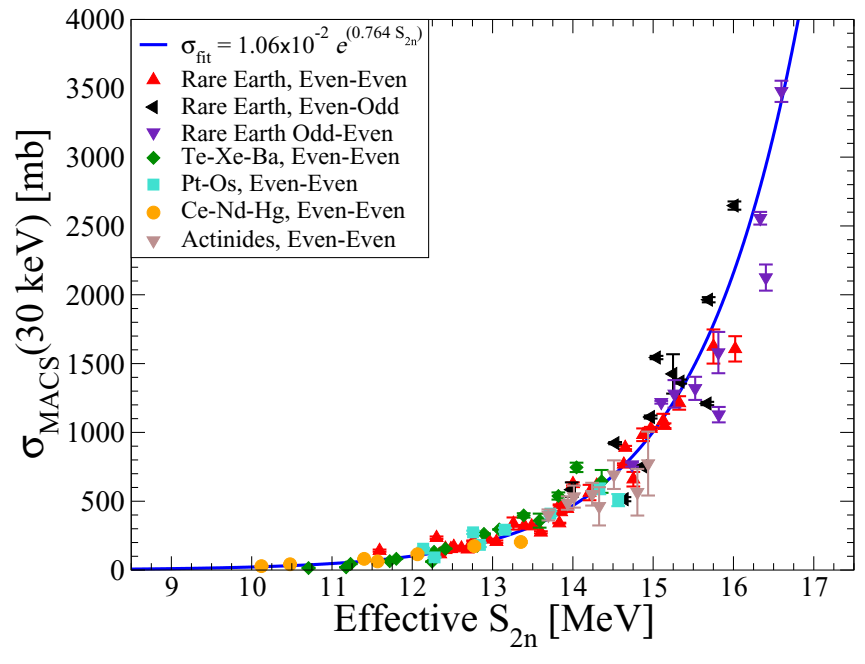

FIG. 3. Shown above are the same 33 even-even, deformed rare earth nuclei together with six other classes. The fit function is the same as in Fig. 1 determined for the original group of 33 deformed rare earth nuclei. As before, for capture on even- $N$ nuclei, we used $S_{2 n}(N+2)$; for capture on odd- $N$ nuclei we used $S_{2 n}(N+1)$, see text. For each class, a single $S_{2 n}$ offset has been introduced to the data, where a negative value $\left(p_{2}<0\right)$ means the actual $S_{2 n}$ was reduced to plot on the figure. The shifts are listed in Table I.

Fig. 1. Thus the data for all these nuclei can be brought into concordance using a single shift constant for each class.

\section{B. Energy dependence}

Finally, we now extend the analysis to include a range of neutron energies. From the KADoNiS database we have cross section data for the neutron energies 5, 10, 15, 20, 25, $30,40,50,60,80$, and $100 \mathrm{keV}$. For each region, the energy dependence of the cross sections was fit by an empirical curve. Using that curve, the data can be normalized to their effective cross section at $30 \mathrm{keV}$. Not surprisingly the energy dependence found is extremely close to a simple $1 / v$ dependence. The dependence differs very slightly from region to region, except for nondeformed nuclei where they deviate somewhat more at neutron energies close to $100 \mathrm{keV}$. To bring all the data into a single correlation plot we apply a single normalization of the form

$$
\sigma_{30}=\sigma_{E_{n}} c_{0}\left(E_{n}\right)^{c_{1}},
$$

where $E_{n}$ is the Maxwell-Boltzmann average energy in keV and $c_{0}$ and $c_{1}$ were fit to to the rare-earth even-even data to give $c_{0}=0.154$ and $c_{1}=0.552$. Note that the corresponding exponent $c_{1}$ for a $1 / v$ dependence would be 0.50 .

Figure 4 now combines all the data for the five mass regions, seven classes of nuclei, and 11 neutron energy distributions from 5-100 keV. This represents over 700 cross-section values, from different nuclear structure regions, masses ranging from $A \approx 110-240$ across the chart of nuclides, exhibiting a simple, analytic connection to nuclear binding energy. There are two remarkable features.

One is simply that the combined data all lie on a reasonably compact trajectory. The other is that the fit function is again 


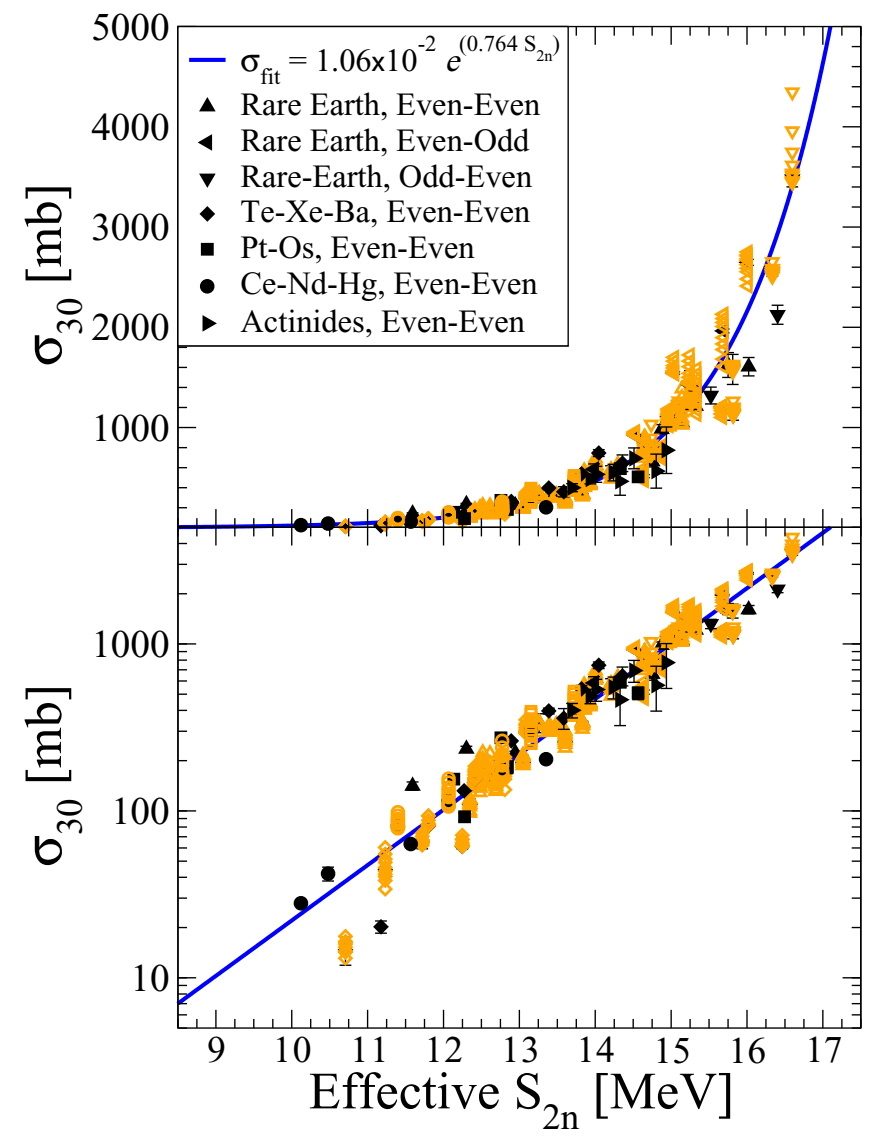

FIG. 4. The figure shows the principal result of this study, the $S_{2 n}$-neutron cross section correlation for all five mass regions, seven classes, and the 11 neutron energy distributions from 5 to $100 \mathrm{keV}$, about 700 data points in total. The fit function is the same as for the original 33 deformed rare earth nuclei at $30 \mathrm{keV}$ in Fig. 1. The cross section $\sigma_{30}$ is the energy-corrected, $30 \mathrm{keV}$ equivalent MACS, as defined in Eq. (3). The $30 \mathrm{keV}$ values are in filled, black symbols while the energy-corrected values are in orange, open symbols. In the upper panel, the cross sections are shown on a linear scale while the lower panel shows the same data, over the identical value range, on a logarithmic scale. The two plots give complementary perspectives on the quality of agreement, especially at high and low cross sections and $S_{2 n}$ values.

the same exponential as that originally used in Fig. 1. That is, the correlation, with 700 data points, covering 97 nuclei, comprising the energy-and-region-combined data, is closely matched by the same exponential as was used for the 33 data points for the even-even deformed rare earth nuclei at neutron energy of $30 \mathrm{keV}$ in Fig. 1(b).

\section{DISCUSSION}

\section{A. Physics underpinning the correlation}

Although the choice of viewing cross sections versus $S_{2 n}$ is simply because it works, it is not without some rationale. That is, why does such a simple ansatz work at all? First, it is well known that the evolution of $S_{2 n}$ is well correlated with other properties of nuclei, such as ratios of certain energy levels or transition rates because it is, in general, correlated with structural changes in nuclei.

More directly relevant to the present subject, elements of the answer recognize that neutron capture in heavy nuclei depends on the overlap of the neutron plus target with the capture state and on the electromagnetic matrix elements connecting the state to lower lying states. Larger $S_{2 n}$ values correspond to higher capture state energies, where the level density is higher, thereby increasing both the number of accessible capture states and the decay phase space, and thus the cross sections.

The same perspective makes it easy to provide a qualitative interpretation of the $S_{2 n}$ shifts in terms of different level densities at the capture state energy. For example, if the even-even actinide nuclei, for which the level density at the capture state is very high (higher degeneracy of shell model configurations), are plotted at a higher effective $S_{2 n}$ they lie close to the rare earth correlation in Fig. 1(b). Likewise, if we also plot the data for the even-odd deformed nuclei (which have higher level density at a given excitation energy due to the absence of one pairing interaction), at a higher effective $S_{2 n}$ the correlation again coincides closely with the even-even rare earth nuclei. As a last example, for spherical nuclei with lower level densities at a given $S_{2 n}$ than deformed nuclei, a decrease in effective $S_{2 n}$ brings these data into better alignment with the deformed rare earth region. This offers an intuitive rationale for the shifts introduced for each region.

\section{B. Predictions of unknown cross sections}

There are a number of implications of the extended correlation, some of considerable importance for $r$-process astrophysics. Some of these were pointed out in the restricted context of Ref. [22] as well but now apply to a much broader set of cross section data. In discussing these we note that reaction networks of neutron capture cross sections usually require input for all $Z$ values including many even-even, even-odd, odd-even, and odd-odd nuclei with unknown cross sections. Even though the data for odd- $A$ nuclei are very limited, we will see that they do allow a wide range of predictions.

\section{Predictions by interpolation}

Since $S_{2 n}$ is determined from the nuclear mass, there is already a wealth of reliable $S_{2 n}$ data, extending to $N$ and $Z$ ranges far beyond known capture cross sections. These allow cross section estimates over a wider range of nuclei than can be measured. Especially intriguing is that it often happens that the $S_{2 n}$ values for nuclei with unknown neutron cross sections are within the range of known values in Figs. 1(b), 3, and 4. Therefore, new cross section estimates can often be made by the more reliable process of interpolation than extrapolation.

To make this statement concrete and to provide a set of specific predictions we have surveyed all even-even, even-odd, odd-even, and odd-odd nuclei in the five regions comprised in Figs. 1, 3, and 4 for all nuclei whose shifted $S_{2 n}$ values fall between $10 \mathrm{MeV}$ and $16 \mathrm{MeV}$.

For each region we took the empirical $S_{2 n}$ values and their shifts as given in Table I. The establishment of shifts for odd $-N$ and odd- $Z$ nuclei (see above) gives at least a plausible approach to regions of nuclei devoid of any cross section data. 
Since the shifts for odd- $N$ and for odd- $Z$ nuclei reflect the physics of pairing, we can assume that similar shifts apply to other regions of odd- $N$ or odd- $Z$ relative to the even-even nuclei in each region. For the former, we simply use the shifts established for the rare earth region of $1.04 \mathrm{MeV}$ and for the latter we use a shift of $1.74 \mathrm{MeV}$ found for the odd- $Z$ rare earth region. Finally, for odd-odd nuclei we use the sum of these shifts, namely $1.04+1.74=2.78 \mathrm{MeV}$ for odd-odd nuclei. Of course, for unknown regions, the uncertainty in the shifts may be quite large, but, even if one allows uncertainties of several hundred $\mathrm{keV}$, the accuracy in predicted cross sections from the $S_{2 n}$ correlation should be superior to existing theoretical methods.

The results are shown in Table II. In all, in this way, we have estimated the cross sections, at a Maxwellian neutron energy distribution of $30 \mathrm{keV}$, solely by interpolation, for 212 nuclei with currently unknown cross sections. Predictions for other neutron energies can be made by using the energy dependence function discussed in Sec. IV B.

Figure 5 gives an impression of the locus and range of these nuclei with colored boxes for those nuclei given in Table II whose cross sections can be predicted by interpolation using solely the fit function of Fig. 1(b).

It is possible to test these estimated shifts in a few cases. Despite the relative scarcity of neutron capture data on odd- $Z$ nuclei, neutron capture cross sections at $30 \mathrm{keV}$ are known for ${ }^{127,129} \mathrm{I}$ and ${ }^{133,135} \mathrm{Cs}$, which lie in the Te-Xe-Ba region, and ${ }^{154} \mathrm{Eu}$ and ${ }^{176} \mathrm{Lu}$, which lie in the rare earth region. For I and Cs, for example, we applied the shifts for odd- $Z$ (an effective $S_{2 n}$ increase) and for the Te-Xe-Ba region (an effective $S_{2 n}$ decrease). As can be seen in Fig. 6, which repeats Fig. 3 with these data superposed with large symbols, the cross sections are in reasonable agreement with the correlation and with the fit function. A similar result is observed in the figure for ${ }^{154} \mathrm{Eu}$ and ${ }^{176} \mathrm{Lu}$, where both of the shifts effectively increase $S_{2 n}$. This lends confidence to predictions in the region of interpolation.

The nuclei in Table II have already known $S_{2 n}$ values with effective $S_{2 n}$ lying between 10 and $16 \mathrm{MeV}$. For some applications involving nuclei further from stability, the $S_{2 n}$ values may not be currently known. However, new measurements of $S_{2 n}$ values in exotic nuclei are typically far easier than a neutron capture measurement. Indeed, in nuclei very far from stability, new $S_{2 n}$ values will often be among the first quantities measured. Whether the masses of new nuclei are measured directly or via the energetics of the reactions used to produce them, the deduced $S_{2 n}$ values will often have accuracies $<100 \mathrm{keV}$. This will be more than adequate for estimating neutron capture cross sections using the correlation we have developed.

\section{Predictions using extrapolation}

Of course, not all $S_{2 n}$ values for nuclei of importance with unknown cross sections lie within the interpolation region. In such cases, extrapolation is required. The accuracy then depends not only on the shifts but on the (unknown) quality of the exponential fit function outside the region from 10 to $16 \mathrm{MeV}$. Indeed, it may happen that a different functional form
TABLE II. For nuclei with effective $S_{2 n}$ values between 10 and $16 \mathrm{MeV}$ whose cross sections are unknown, estimated cross sections at $k T=30 \mathrm{keV}$ are listed using the fit function in Eq. (2) and the shift values in Table I. An uncertainty of $25 \%$ should be assigned to all predictions based on the cross section reproduction for other nuclei with a similar effective $S_{2 n}$.

\begin{tabular}{|c|c|c|c|}
\hline Isotope & $Z$ & $N$ & $\begin{array}{c}\sigma_{\text {MACS }} \\
\text { (Prediction) }\end{array}$ \\
\hline \multicolumn{4}{|c|}{ Rare earth region } \\
\hline${ }^{149} \mathrm{Nd}$ & 60 & 89 & 311 \\
\hline${ }^{151} \mathrm{Nd}$ & 60 & 91 & 361 \\
\hline${ }^{152} \mathrm{Nd}$ & 60 & 92 & 89 \\
\hline${ }^{153} \mathrm{Nd}$ & 60 & 93 & 197 \\
\hline${ }^{154} \mathrm{Nd}$ & 60 & 94 & 41 \\
\hline${ }^{155} \mathrm{Nd}$ & 60 & 95 & 90 \\
\hline${ }^{151} \mathrm{Pm}$ & 61 & 90 & 1133 \\
\hline${ }^{153} \mathrm{Pm}$ & 61 & 92 & 540 \\
\hline${ }^{154} \mathrm{Pm}$ & 61 & 93 & 1196 \\
\hline${ }^{155} \mathrm{Pm}$ & 61 & 94 & 264 \\
\hline${ }^{156} \mathrm{Pm}$ & 61 & 95 & 585 \\
\hline${ }^{157} \mathrm{Pm}$ & 61 & 96 & 114 \\
\hline${ }^{158} \mathrm{Pm}$ & 61 & 97 & 193 \\
\hline${ }^{151} \mathrm{Sm}$ & 62 & 89 & 934 \\
\hline${ }^{153} \mathrm{Sm}$ & 62 & 91 & 921 \\
\hline${ }^{155} \mathrm{Sm}$ & 62 & 93 & 504 \\
\hline${ }^{156} \mathrm{Sm}$ & 62 & 94 & 105 \\
\hline${ }^{157} \mathrm{Sm}$ & 62 & 95 & 232 \\
\hline${ }^{158} \mathrm{Sm}$ & 62 & 96 & 52 \\
\hline${ }^{159} \mathrm{Sm}$ & 62 & 97 & 116 \\
\hline${ }^{157} \mathrm{Eu}$ & 63 & 94 & 675 \\
\hline${ }^{158} \mathrm{Eu}$ & 63 & 95 & 1495 \\
\hline${ }^{159} \mathrm{Eu}$ & 63 & 96 & 356 \\
\hline${ }^{160} \mathrm{Eu}$ & 63 & 97 & 789 \\
\hline${ }^{161} \mathrm{Eu}$ & 63 & 98 & 159 \\
\hline${ }^{162} \mathrm{Eu}$ & 63 & 99 & 269 \\
\hline${ }^{159} \mathrm{Gd}$ & 64 & 95 & 657 \\
\hline${ }^{161} \mathrm{Gd}$ & 64 & 97 & 327 \\
\hline${ }^{161} \mathrm{~Tb}$ & 65 & 96 & 1028 \\
\hline${ }^{158}$ Dy & 66 & 92 & 1383 \\
\hline${ }^{165} \mathrm{Dy}$ & 66 & 99 & 405 \\
\hline${ }^{166} \mathrm{Dy}$ & 66 & 100 & 112 \\
\hline${ }^{167} \mathrm{Dy}$ & 66 & 101 & 248 \\
\hline${ }^{167} \mathrm{Ho}$ & 67 & 100 & 641 \\
\hline${ }^{168} \mathrm{Ho}$ & 67 & 101 & 1419 \\
\hline${ }^{169} \mathrm{Ho}$ & 67 & 102 & 349 \\
\hline${ }^{170} \mathrm{Ho}$ & 67 & 103 & 590 \\
\hline${ }^{169} \mathrm{Er}$ & 68 & 101 & 593 \\
\hline${ }^{171} \mathrm{Er}$ & 68 & 103 & 336 \\
\hline${ }^{171} \mathrm{Tm}$ & 69 & 102 & 960 \\
\hline${ }^{172} \mathrm{Tm}$ & 69 & 103 & 2127 \\
\hline${ }^{173} \mathrm{Tm}$ & 69 & 104 & 450 \\
\hline${ }^{174} \mathrm{Tm}$ & 69 & 105 & 997 \\
\hline${ }^{175} \mathrm{Yb}$ & 70 & 105 & 383 \\
\hline${ }^{177} \mathrm{Yb}$ & 70 & 107 & 295 \\
\hline${ }^{177} \mathrm{Lu}$ & 71 & 106 & 723 \\
\hline${ }^{178} \mathrm{Lu}$ & 71 & 107 & 1602 \\
\hline${ }^{179} \mathrm{Lu}$ & 71 & 108 & 354 \\
\hline${ }^{180} \mathrm{Lu}$ & 71 & 109 & 783 \\
\hline${ }^{181} \mathrm{Lu}$ & 71 & 110 & 189 \\
\hline${ }^{182} \mathrm{Lu}$ & 71 & 111 & 418 \\
\hline
\end{tabular}


TABLE II. (Continued.)

\begin{tabular}{|c|c|c|c|}
\hline Isotope & $Z$ & $N$ & $\begin{array}{c}\sigma_{\text {MACS }} \\
\text { (Prediction) }\end{array}$ \\
\hline${ }^{172} \mathrm{Hf}$ & 72 & 100 & 1583 \\
\hline${ }^{175} \mathrm{Hf}$ & 72 & 103 & 2037 \\
\hline${ }^{181} \mathrm{Hf}$ & 72 & 109 & 310 \\
\hline${ }^{183} \mathrm{Hf}$ & 72 & 111 & 166 \\
\hline${ }^{184} \mathrm{Hf}$ & 72 & 112 & 50 \\
\hline${ }^{185} \mathrm{Hf}$ & 72 & 113 & 111 \\
\hline${ }^{179} \mathrm{Ta}$ & 73 & 106 & 2119 \\
\hline${ }^{181} \mathrm{Ta}$ & 73 & 108 & 830 \\
\hline${ }^{182} \mathrm{Ta}$ & 73 & 109 & 1838 \\
\hline${ }^{183} \mathrm{Ta}$ & 73 & 110 & 467 \\
\hline${ }^{184} \mathrm{Ta}$ & 73 & 111 & 789 \\
\hline${ }^{185} \mathrm{Ta}$ & 73 & 112 & 295 \\
\hline${ }^{186} \mathrm{Ta}$ & 73 & 113 & 499 \\
\hline${ }^{176} \mathrm{~W}$ & 74 & 102 & 2027 \\
\hline${ }^{178} \mathrm{~W}$ & 74 & 104 & 1345 \\
\hline${ }^{181} \mathrm{~W}$ & 74 & 107 & 1856 \\
\hline${ }^{187} \mathrm{~W}$ & 74 & 113 & 285 \\
\hline${ }^{188} \mathrm{~W}$ & 74 & 114 & 92 \\
\hline${ }^{189} \mathrm{~W}$ & 74 & 115 & 203 \\
\hline \multicolumn{4}{|c|}{ Te-Xe-Ba region } \\
\hline${ }^{116} \mathrm{Te}$ & 52 & 64 & 1272 \\
\hline${ }^{118} \mathrm{Te}$ & 52 & 66 & 714 \\
\hline${ }^{119} \mathrm{Te}$ & 52 & 67 & 1581 \\
\hline${ }^{121} \mathrm{Te}$ & 52 & 69 & 908 \\
\hline${ }^{123} \mathrm{Te}$ & 52 & 71 & 518 \\
\hline${ }^{125} \mathrm{Te}$ & 52 & 73 & 310 \\
\hline${ }^{127} \mathrm{Te}$ & 52 & 75 & 194 \\
\hline${ }^{129} \mathrm{Te}$ & 52 & 77 & 126 \\
\hline${ }^{131} \mathrm{Te}$ & 52 & 79 & 84 \\
\hline${ }^{132} \mathrm{Te}$ & 52 & 80 & 26 \\
\hline${ }^{123} \mathrm{I}$ & 53 & 70 & 1492 \\
\hline${ }^{125} \mathrm{I}$ & 53 & 72 & 843 \\
\hline${ }^{126} \mathrm{I}$ & 53 & 73 & 1867 \\
\hline${ }^{127} \mathrm{I}$ & 53 & 74 & 523 \\
\hline${ }^{128} \mathrm{I}$ & 53 & 75 & 1159 \\
\hline${ }^{129} \mathrm{I}$ & 53 & 76 & 334 \\
\hline${ }^{130} \mathrm{I}$ & 53 & 77 & 740 \\
\hline${ }^{131} \mathrm{I}$ & 53 & 78 & 224 \\
\hline${ }^{132} \mathrm{I}$ & 53 & 79 & 497 \\
\hline${ }^{133} \mathrm{I}$ & 53 & 80 & 154 \\
\hline${ }^{122} \mathrm{Xe}$ & 54 & 68 & 1160 \\
\hline${ }^{125} \mathrm{Xe}$ & 54 & 71 & 1372 \\
\hline${ }^{127} \mathrm{Xe}$ & 54 & 73 & 760 \\
\hline${ }^{129} \mathrm{Xe}$ & 54 & 75 & 447 \\
\hline${ }^{131} \mathrm{Xe}$ & 54 & 77 & 278 \\
\hline${ }^{133} \mathrm{Xe}$ & 54 & 79 & 183 \\
\hline${ }^{127} \mathrm{Cs}$ & 55 & 72 & 1973 \\
\hline${ }^{129} \mathrm{Cs}$ & 55 & 74 & 1156 \\
\hline${ }^{131} \mathrm{Cs}$ & 55 & 76 & 761 \\
\hline${ }^{132} \mathrm{Cs}$ & 55 & 77 & 1685 \\
\hline${ }^{133} \mathrm{Cs}$ & 55 & 78 & 518 \\
\hline${ }^{134} \mathrm{Cs}$ & 55 & 79 & 1148 \\
\hline${ }^{135} \mathrm{Cs}$ & 55 & 80 & 341 \\
\hline${ }^{126} \mathrm{Ba}$ & 56 & 70 & 1577 \\
\hline
\end{tabular}

TABLE II. (Continued.)

\begin{tabular}{|c|c|c|c|}
\hline Isotope & $Z$ & $N$ & $\begin{array}{c}\sigma_{\mathrm{MACS}} \\
\text { (Prediction) }\end{array}$ \\
\hline${ }^{128} \mathrm{Ba}$ & 56 & 72 & 839 \\
\hline${ }^{129} \mathrm{Ba}$ & 56 & 73 & 1859 \\
\hline${ }^{131} \mathrm{Ba}$ & 56 & 75 & 1080 \\
\hline${ }^{133} \mathrm{Ba}$ & 56 & 77 & 653 \\
\hline${ }^{135} \mathrm{Ba}$ & 56 & 79 & 420 \\
\hline \multicolumn{4}{|c|}{ Ce-Nd-Hg region } \\
\hline${ }^{143} \mathrm{Ce}$ & 58 & 85 & 54 \\
\hline${ }^{145} \mathrm{Ce}$ & 58 & 87 & 32 \\
\hline${ }^{143} \operatorname{Pr}$ & 59 & 84 & 153 \\
\hline${ }^{144} \mathrm{Pr}$ & 59 & 85 & 338 \\
\hline${ }^{145} \mathrm{Pr}$ & 59 & 86 & 87 \\
\hline${ }^{146} \mathrm{Pr}$ & 59 & 87 & 192 \\
\hline${ }^{145} \mathrm{Nd}$ & 60 & 85 & 143 \\
\hline${ }^{146} \mathrm{Nd}$ & 60 & 86 & 38 \\
\hline${ }^{147} \mathrm{Nd}$ & 60 & 87 & 84 \\
\hline${ }^{188} \mathrm{Hg}$ & 80 & 108 & 1367 \\
\hline${ }^{190} \mathrm{Hg}$ & 80 & 110 & 913 \\
\hline${ }^{191} \mathrm{Hg}$ & 80 & 111 & 2022 \\
\hline${ }^{192} \mathrm{Hg}$ & 80 & 112 & 638 \\
\hline${ }^{193} \mathrm{Hg}$ & 80 & 113 & 1414 \\
\hline${ }^{194} \mathrm{Hg}$ & 80 & 114 & 425 \\
\hline${ }^{195} \mathrm{Hg}$ & 80 & 115 & 942 \\
\hline${ }^{197} \mathrm{Hg}$ & 80 & 117 & 636 \\
\hline${ }^{199} \mathrm{Hg}$ & 80 & 119 & 409 \\
\hline${ }^{201} \mathrm{Hg}$ & 80 & 121 & 238 \\
\hline${ }^{203} \mathrm{Hg}$ & 80 & 123 & 163 \\
\hline \multicolumn{4}{|c|}{ Pt-Os region } \\
\hline${ }^{180}$ Os & 76 & 104 & 1921 \\
\hline${ }^{182}$ Os & 76 & 106 & 1207 \\
\hline${ }^{185}$ Os & 76 & 109 & 1348 \\
\hline${ }^{187}$ Os & 76 & 111 & 846 \\
\hline${ }^{189} \mathrm{Os}$ & 76 & 113 & 548 \\
\hline${ }^{191}$ Os & 76 & 115 & 405 \\
\hline${ }^{193}$ Os & 76 & 117 & 252 \\
\hline${ }^{194}$ Os & 76 & 118 & 66 \\
\hline${ }^{195}$ Os & 76 & 119 & 146 \\
\hline${ }^{189} \mathrm{Ir}$ & 77 & 112 & 1587 \\
\hline${ }^{191} \mathrm{Ir}$ & 77 & 114 & 1141 \\
\hline${ }^{193} \mathrm{Ir}$ & 77 & 116 & 683 \\
\hline${ }^{194} \mathrm{Ir}$ & 77 & 117 & 1513 \\
\hline${ }^{195} \mathrm{Ir}$ & 77 & 118 & 437 \\
\hline${ }^{196} \mathrm{Ir}$ & 77 & 119 & 968 \\
\hline${ }^{197} \mathrm{Ir}$ & 77 & 120 & 313 \\
\hline${ }^{198} \mathrm{Ir}$ & 77 & 121 & 693 \\
\hline${ }^{186} \mathrm{Pt}$ & 78 & 108 & 1533 \\
\hline${ }^{188} \mathrm{Pt}$ & 78 & 110 & 1070 \\
\hline${ }^{191} \mathrm{Pt}$ & 78 & 113 & 1613 \\
\hline${ }^{192} \mathrm{Pt}$ & 78 & 114 & 493 \\
\hline${ }^{193} \mathrm{Pt}$ & 78 & 115 & 1092 \\
\hline${ }^{194} \mathrm{Pt}$ & 78 & 116 & 315 \\
\hline${ }^{195} \mathrm{Pt}$ & 78 & 117 & 697 \\
\hline${ }^{197} \mathrm{Pt}$ & 78 & 119 & 432 \\
\hline${ }^{199} \mathrm{Pt}$ & 78 & 121 & 281 \\
\hline${ }^{200} \mathrm{Pt}$ & 78 & 122 & 80 \\
\hline
\end{tabular}


TABLE II. (Continued.)

\begin{tabular}{|c|c|c|c|}
\hline Isotope & $Z$ & $N$ & $\begin{array}{c}\sigma_{\mathrm{MACS}} \\
\text { (Prediction) }\end{array}$ \\
\hline $\begin{array}{l}{ }^{201} \mathrm{Pt} \\
\text { Actinides }\end{array}$ & 78 & 123 & 177 \\
\hline${ }^{224} \mathrm{Rn}$ & 86 & 138 & 188 \\
\hline${ }^{225} \mathrm{Rn}$ & 86 & 139 & 416 \\
\hline${ }^{226} \mathrm{Rn}$ & 86 & 140 & 162 \\
\hline${ }^{227} \mathrm{Rn}$ & 86 & 141 & 359 \\
\hline${ }^{222} \mathrm{Ra}$ & 88 & 134 & 742 \\
\hline${ }^{223} \mathrm{Ra}$ & 88 & 135 & 1644 \\
\hline${ }^{224} \mathrm{Ra}$ & 88 & 136 & 574 \\
\hline${ }^{225} \mathrm{Ra}$ & 88 & 137 & 1271 \\
\hline${ }^{226} \mathrm{Ra}$ & 88 & 138 & 413 \\
\hline${ }^{227} \mathrm{Ra}$ & 88 & 139 & 915 \\
\hline${ }^{228} \mathrm{Ra}$ & 88 & 140 & 327 \\
\hline${ }^{229} \mathrm{Ra}$ & 88 & 141 & 725 \\
\hline${ }^{230} \mathrm{Ra}$ & 88 & 142 & 240 \\
\hline${ }^{231} \mathrm{Ra}$ & 88 & 143 & 532 \\
\hline${ }^{232} \mathrm{Ra}$ & 88 & 144 & 170 \\
\hline${ }^{233} \mathrm{Ra}$ & 88 & 145 & 377 \\
\hline${ }^{229} \mathrm{Ac}$ & 89 & 140 & 1823 \\
\hline${ }^{231} \mathrm{Ac}$ & 89 & 142 & 1270 \\
\hline${ }^{233} \mathrm{Ac}$ & 89 & 144 & 864 \\
\hline${ }^{234} \mathrm{Ac}$ & 89 & 145 & 1914 \\
\hline${ }^{224} \mathrm{Th}$ & 90 & 134 & 2008 \\
\hline${ }^{226} \mathrm{Th}$ & 90 & 136 & 1514 \\
\hline${ }^{228} \mathrm{Th}$ & 90 & 138 & 1019 \\
\hline${ }^{230} \mathrm{Th}$ & 90 & 140 & 699 \\
\hline${ }^{231} \mathrm{Th}$ & 90 & 141 & 1548 \\
\hline${ }^{233} \mathrm{Th}$ & 90 & 143 & 992 \\
\hline${ }^{234} \mathrm{Th}$ & 90 & 144 & 311 \\
\hline${ }^{235} \mathrm{Th}$ & 90 & 145 & 690 \\
\hline${ }^{235} \mathrm{~Pa}$ & 91 & 144 & 1605 \\
\hline${ }^{232} \mathrm{U}$ & 92 & 140 & 1558 \\
\hline${ }^{235} \mathrm{U}$ & 92 & 143 & 1924 \\
\hline${ }^{237} \mathrm{U}$ & 92 & 145 & 1251 \\
\hline${ }^{239} \mathrm{U}$ & 92 & 147 & 825 \\
\hline${ }^{239} \mathrm{~Np}$ & 93 & 146 & 2003 \\
\hline${ }^{236} \mathrm{Pu}$ & 94 & 142 & 1921 \\
\hline${ }^{238} \mathrm{Pu}$ & 94 & 144 & 1124 \\
\hline${ }^{241} \mathrm{Pu}$ & 94 & 147 & 1540 \\
\hline${ }^{243} \mathrm{Pu}$ & 94 & 149 & 1053 \\
\hline${ }^{245} \mathrm{Pu}$ & 94 & 151 & 718 \\
\hline${ }^{242} \mathrm{Cm}$ & 96 & 146 & 1430 \\
\hline${ }^{245} \mathrm{Cm}$ & 96 & 149 & 2133 \\
\hline${ }^{247} \mathrm{Cm}$ & 96 & 151 & 1337 \\
\hline${ }^{248} \mathrm{Cm}$ & 96 & 152 & 322 \\
\hline${ }^{249} \mathrm{Cm}$ & 96 & 153 & 714 \\
\hline${ }^{249} \mathrm{Bk}$ & 97 & 152 & 1439 \\
\hline${ }^{246} \mathrm{Cf}$ & 98 & 148 & 2096 \\
\hline${ }^{248} \mathrm{Cf}$ & 98 & 150 & 1150 \\
\hline${ }^{250} \mathrm{Cf}$ & 98 & 152 & 564 \\
\hline${ }^{251} \mathrm{Cf}$ & 98 & 153 & 1250 \\
\hline${ }^{252} \mathrm{Cf}$ & 98 & 154 & 402 \\
\hline${ }^{253} \mathrm{Cf}$ & 98 & 155 & 891 \\
\hline${ }^{253}$ Es & 99 & 154 & 1814 \\
\hline
\end{tabular}

TABLE II. (Continued.)

\begin{tabular}{lccc}
\hline \hline Isotope & $Z$ & $N$ & $\begin{array}{c}\sigma_{\text {MACS }} \\
\text { (Prediction) }\end{array}$ \\
${ }^{252} \mathrm{Fm}$ & 100 & 152 & 1022 \\
${ }^{254} \mathrm{Fm}$ & 100 & 154 & 699 \\
${ }^{255} \mathrm{Fm}$ & 100 & 155 & 1548 \\
\hline \hline
\end{tabular}

will be preferred (such as the power law used in Ref. [22]). However, if only one or two neutron capture measurements can be made for nuclei with low $S_{2 n}$ values, it should be possible to better define the entire correlation down to such values. Such measurements, though difficult, might well be worth the effort.

\section{Vetting the predictions for new regions}

If a particular nucleus in an unstudied or poorly known region is of high significance, but particularly intractable experimentally, the correlation provides an alternate approach to estimating its neutron capture cross section that may be much easier. Less challenging measurements can be performed on a couple of neighboring nuclei to determine the correlation for that region, and the challenging cross section may then be inferred without the need for an extremely difficult or impossible measurement on that particular nucleus. For example, a few key accurate measurements of neutron capture cross sections in odd-even isotopes outside the rare earth region, even at a single neutron energy, would be valuable to refine their correlations and the assumed shifts and allow the prediction of numerous other cross sections.

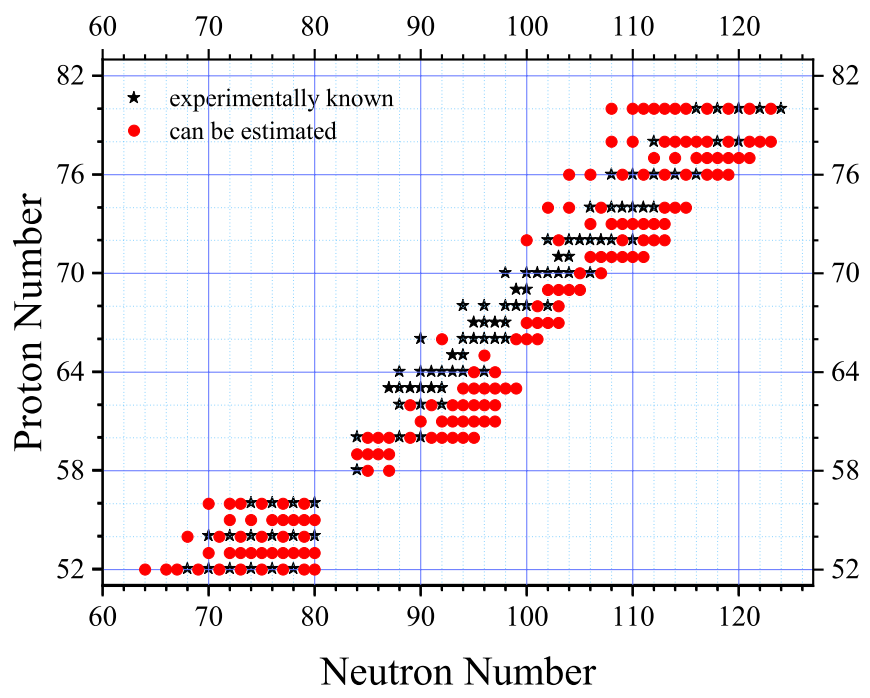

FIG. 5. Chart of the nuclides from $\mathrm{Te}$ to $\mathrm{Pb}$ with colored boxes for the nuclei in Table II whose neutron capture cross sections can be estimated from Fig. 4 by interpolation using the fit function of Fig. 1(b). Table II also includes predictions for more than 50 actinide nuclei outside this range that are not shown above. 


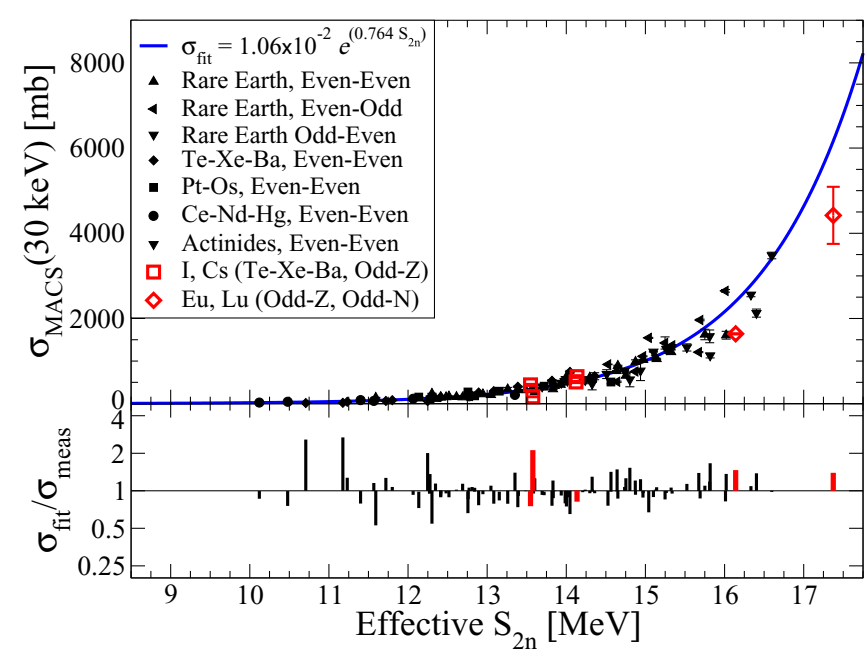

FIG. 6. Same as Fig. 3 except for the superposition of six nuclei (with large symbols) with known cross sections in regions where a specific shift had not been determined, but was rather extrapolated from the existing shifts. The quality of agreement is comparable to the nuclei fit in the study.

\section{Caveat}

A limitation of this approach is that the correlations fail below about mass $A \approx 110$. The likely reason is that these lighter nuclei have low level densities such that even Maxwellian averaged neutron capture reactions do not average over a sufficient number of levels (resonances) in the capture state region so that the resulting cross sections are dominated by one or a few resonances and show nonstatistical behavior.

\section{CONCLUSIONS}

In the paper we have extended the neutron capture cross section versus $S_{2 n}$ correlation to a wider range of medium mass and heavy nuclei and to a range of neutron energies from 5-100 keV, important to the $r$ process and to other applications. We have shown that a single compact correlation (well matched by a simple, physically motivated, exponential function) accommodates this wide array of data, and that the approach provides a way to predict unknown cross sections. In many cases these predictions can be made by interpolation where the expected accuracy is particularly high. In this vein, we provided detailed predictions for over 200 nuclei with currently unknown cross sections. An obvious further step is to use these cross section estimates to refine nucleosynthesis calculations of the $r$ process.

\section{ACKNOWLEDGMENTS}

We thank M. R. Mumpower for helpful discussions regarding $r$-process abundance predictions and cross section impacts. A.C. and R.F.C. were supported by the U.S. Department of Energy through the Los Alamos National Laboratory. Los Alamos National Laboratory is operated by Triad National Security, LLC, for the National Nuclear Security Administration of U.S. Department of Energy (Contract No. 89233218CNA000001). R.B.C. acknowledges support from the Max-Planck Partner group.

\section{APPENDIX: DOCUMENTATION OF DATA SOURCES AND VALUES}

We report the nuclei with their respective $S_{2 n}$ values and cross sections and associated uncertainties for the studies performed as part of this work in Tables III-X. The cross sections and uncertainties are taken from Refs. [23,26,27] while $S_{2 n}$ values are taken from Ref. [24].

TABLE III. Data from Refs. [23,24] for Te-Xe-Ba even-even transitional nuclei.

Te-Xe-Ba even-even (transitional $52 \leqslant Z \leqslant 56,50 \leqslant N \leqslant 82$ )

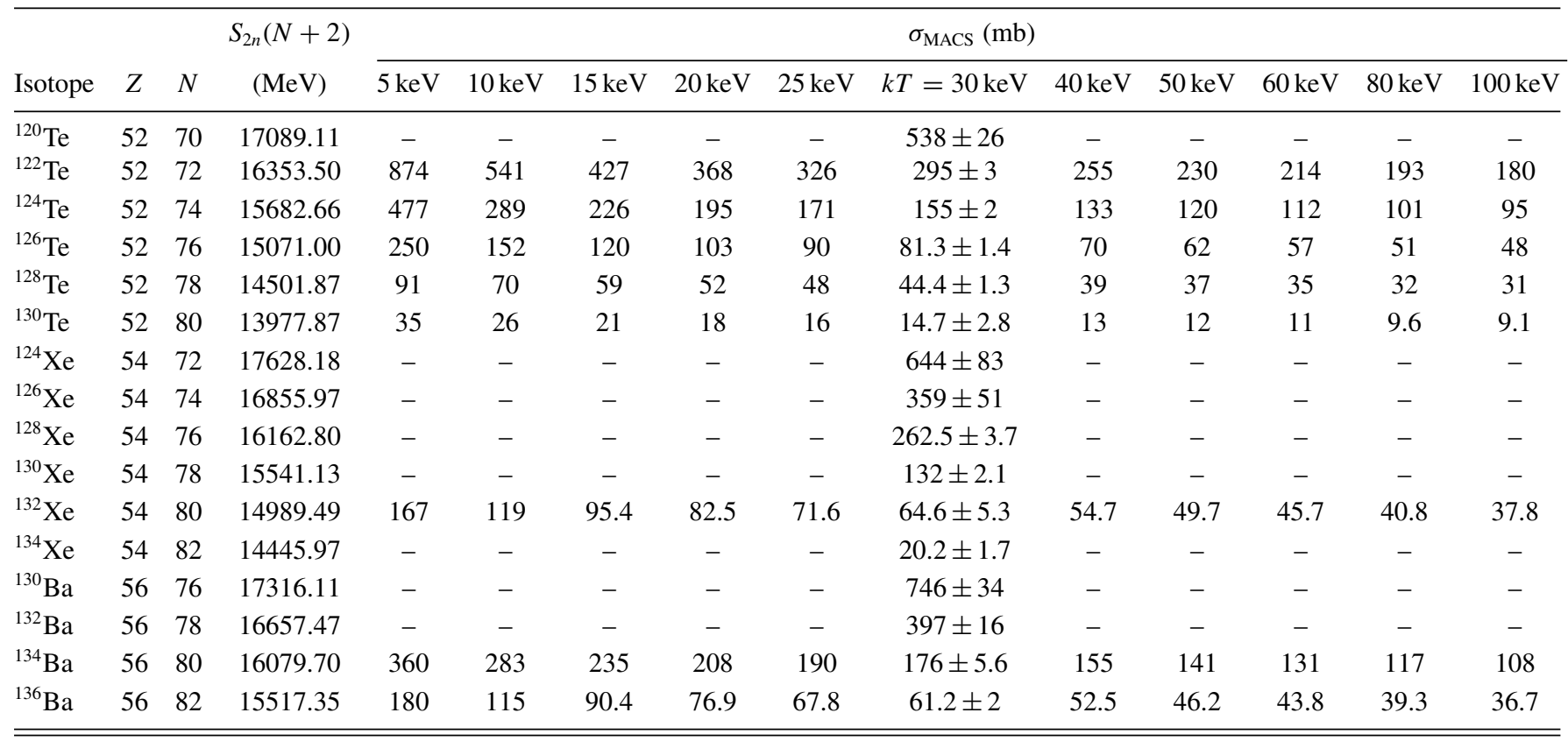


TABLE IV. Data from Refs. [23,24] for rare-earth, even-even nuclei.

Rare earth even-even (deformed $60 \leqslant Z \leqslant 74,88 \leqslant N \leqslant 112$ )

$$
S_{2 n}(N+2) \quad \sigma_{\text {MACS }}(\mathrm{mb})
$$

$\begin{array}{llllllllllllllll}\text { Isotope } & Z & N & (\mathrm{MeV}) & 5 \mathrm{keV} & 10 \mathrm{keV} & 15 \mathrm{keV} & 20 \mathrm{keV} & 25 \mathrm{keV} & k T=30 \mathrm{keV} & 40 \mathrm{keV} & 50 \mathrm{keV} & 60 \mathrm{keV} & 80 \mathrm{keV} & 100 \mathrm{keV}\end{array}$

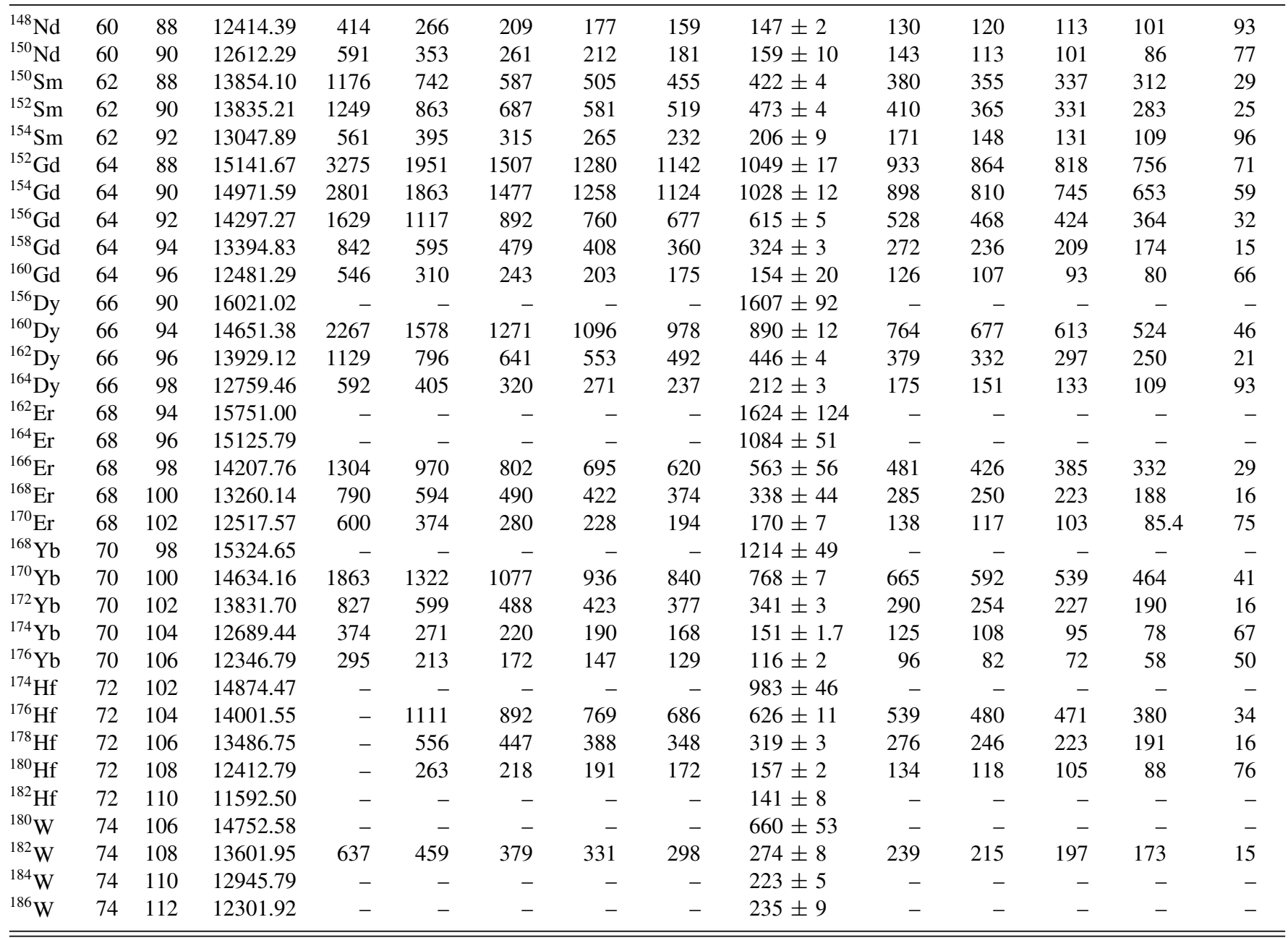

TABLE V. Data from Refs. [23,24] for rare-earth, even-odd nuclei.

Rare earth even-odd (deformed $60 \leqslant Z \leqslant 74,88 \leqslant N \leqslant 112$ )

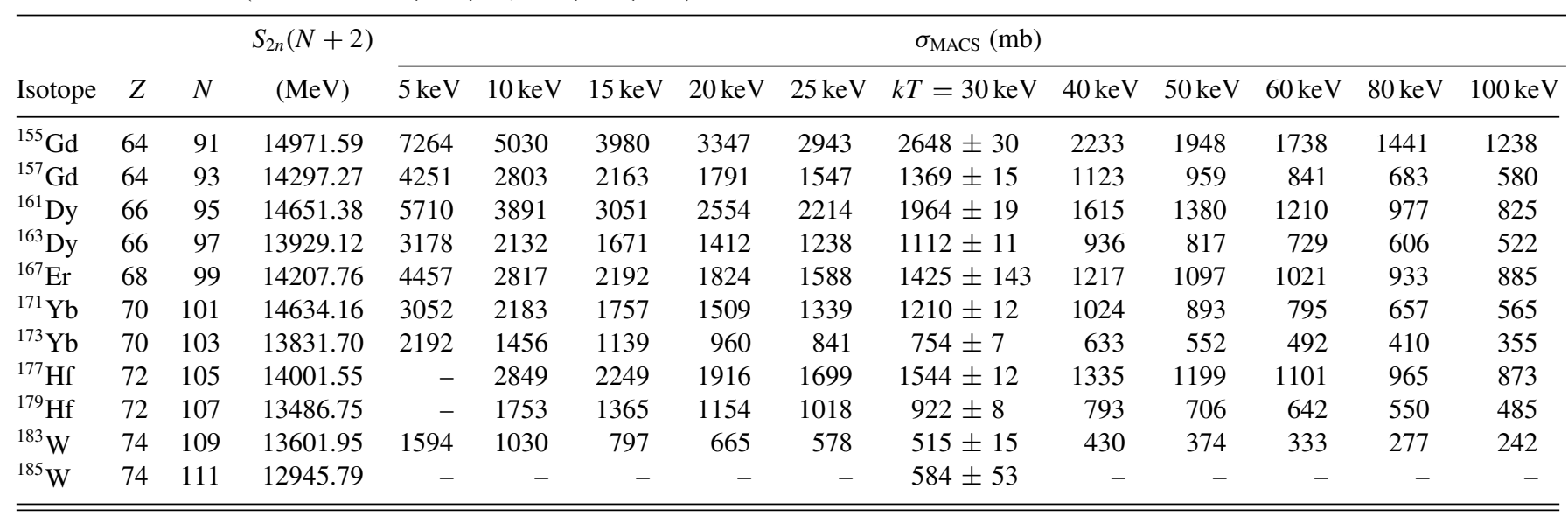


TABLE VI. Data from Refs. [23,24] for rare-earth, odd-even nuclei.

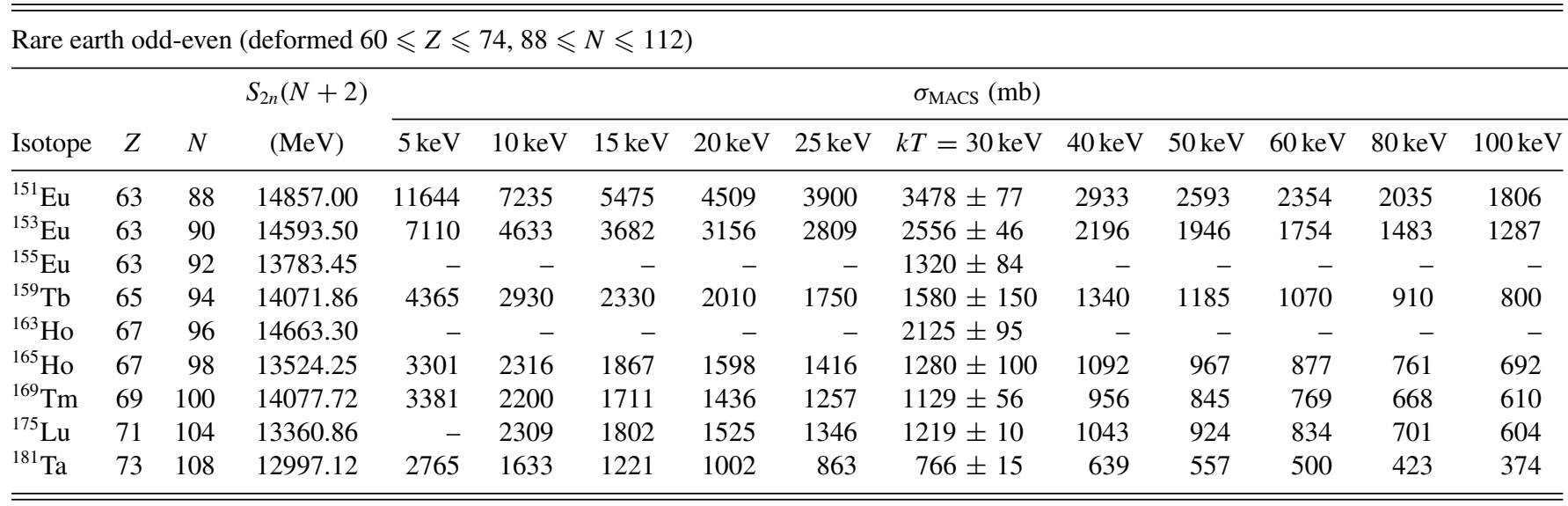

TABLE VII. Data from Refs. [23,24] for Pt-Os $\gamma$-soft, even-even nuclei.

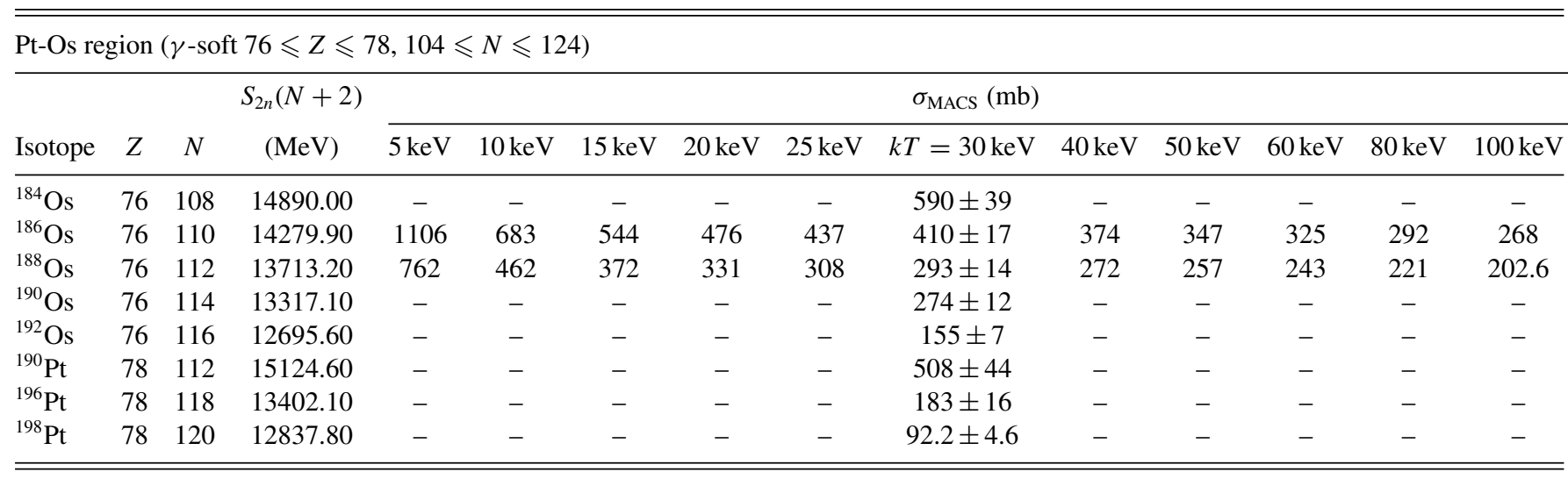

TABLE VIII. Data from Refs. [23,24] for Ce-Nd-Hg spherical, even-even nuclei.

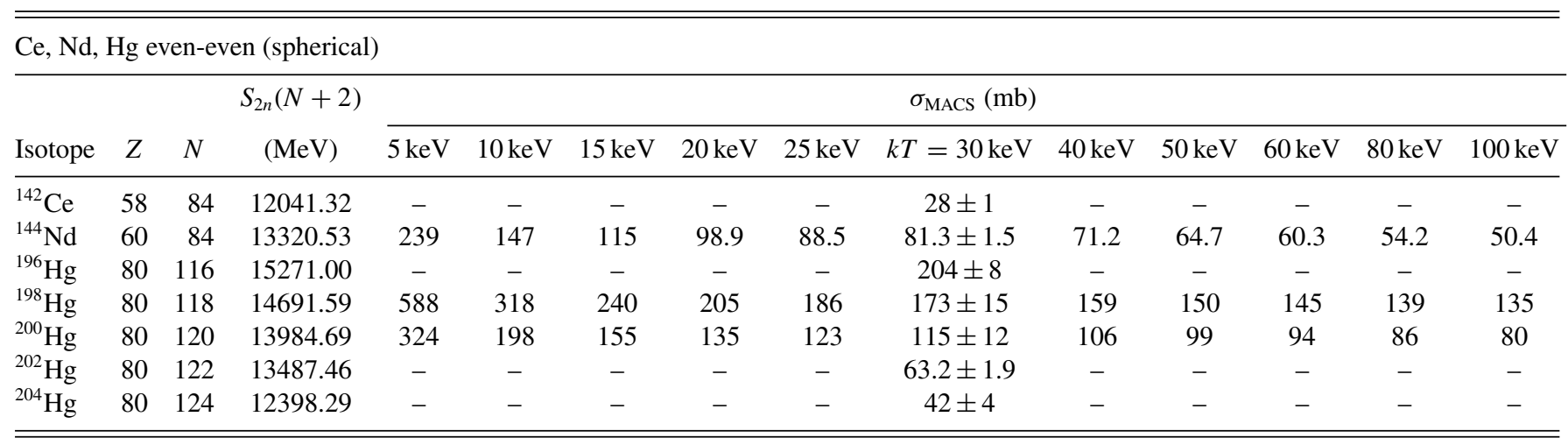


TABLE IX. Data from Refs. [24,26,27] for actinide, even-even nuclei. While energy dependent cross sections can be calculated with ENDF/B-VII.1 and the NucRates, because the evaluation process is different than for KADoNiS, they were not used for comparison and are not reported here.

Actinide even-even (prolate deformed $82 \leqslant 126,126 \leqslant N$ )

\begin{tabular}{|c|c|c|c|c|c|c|c|c|c|c|c|c|c|c|}
\hline Isotope & $Z$ & $N$ & $\begin{array}{c}S_{2 n}(N+2) \\
(\mathrm{MeV})\end{array}$ & \multicolumn{11}{|c|}{$\sigma_{\mathrm{MACS}}(\mathrm{mb})$} \\
\hline${ }^{232} \mathrm{Th}$ & 90 & 142 & 10976.41 & - & - & - & - & - & $487 \pm 49$ & - & - & - & - & - \\
\hline${ }^{236} \mathrm{U}$ & 92 & 144 & 11279.50 & - & - & - & - & - & $551 \pm 83$ & - & - & - & - & - \\
\hline${ }^{238} \mathrm{U}$ & 92 & 146 & 10734.91 & - & - & - & - & - & $400 \pm 40$ & - & - & - & - & - \\
\hline${ }^{244} \mathrm{Cm}$ & 96 & 148 & 11977.56 & - & - & - & - & - & $774 \pm 232$ & - & - & - & - & - \\
\hline${ }^{246} \mathrm{Cm}$ & 96 & 150 & 11366.85 & - & - & - & - & - & $463 \pm 139$ & - & - & - & - & - \\
\hline
\end{tabular}

TABLE X. Data from Refs. [23,24] for I, Cs, Eu, and Lu isotopes which were compared to predictions.

Cross sections for predicted isotopes (see Discussion in Sec. V B 1)

\begin{tabular}{|c|c|c|c|c|c|c|c|c|c|c|c|c|c|c|}
\hline \multirow[b]{2}{*}{ Isotope } & \multirow[b]{2}{*}{$Z$} & \multirow[b]{2}{*}{$N$} & \multirow{2}{*}{$\begin{array}{c}S_{2 n}(N+2) \\
(\mathrm{MeV})\end{array}$} & \multicolumn{11}{|c|}{$\sigma_{\mathrm{MACS}}(\mathrm{mb})$} \\
\hline & & & & $5 \mathrm{keV}$ & $10 \mathrm{keV}$ & $15 \mathrm{keV}$ & $20 \mathrm{keV}$ & $25 \mathrm{keV}$ & $k T=30 \mathrm{keV}$ & $40 \mathrm{keV}$ & $50 \mathrm{keV}$ & $60 \mathrm{keV}$ & $80 \mathrm{keV}$ & $100 \mathrm{keV}$ \\
\hline${ }^{127} \mathrm{I}$ & 53 & 74 & 15665.85 & - & - & - & - & - & $635 \pm 3$ & - & - & - & - & - \\
\hline${ }^{133} \mathrm{Cs}$ & 55 & 78 & 15653.35 & - & - & - & - & - & $509 \pm 2$ & - & - & - & - & - \\
\hline${ }^{135} \mathrm{Cs}$ & 55 & 80 & 15106.60 & - & - & - & - & - & $160 \pm 1$ & - & - & - & - & - \\
\hline${ }^{154} \mathrm{Eu}$ & 63 & 91 & 14593.50 & - & - & - & - & - & $4420 \pm 670$ & - & - & - & - & - \\
\hline
\end{tabular}

[1] A. S. Eddington, The Observatory 43, 341 (1920).

[2] A. G. W. Cameron, Annu. Rev. Nucl. Part. Sci. 8, 299 (1958).

[3] E. M. Burbidge, G. R. Burbidge, W. A. Fowler, and F. Hoyle, Rev. Mod. Phys. 29, 547 (1957).

[4] J. J. Cowan and W. K. Rose, Astrophys. J. 212, 149 (1977).

[5] F. Herwig, M. Pignatari, P. R. Woodward, D. H. Porter, G. Rockefeller, C. L. Fryer, M. Bennett, and R. Hirschi, Astrophys. J. 727, 89 (2011).

[6] M. R. Mumpower, R. Surman, G. C. McLaughlin, and A. Aprahamian, Prog. Part. Nucl. Phys. 86, 86 (2016).

[7] K.-L. Kratz, J.-P. Bitouzet, F.-K. Thielemann, P. Moeller, and B. Pfeiffer, Astrophys. J. 403, 216 (1993).

[8] K. Farouqi, K. Kratz, B. Pfeiffer, T. Rauscher, F. Thielemann, and J. W. Truran, Astrophys. J. 712, 1359 (2010).

[9] R. Surman, J. Beun, G. C. McLaughlin, and W. R. Hix, Phys. Rev. C 79, 045809 (2009).

[10] M. R. Mumpower, G. C. McLaughlin, and R. Surman, Phys. Rev. C 86, 035803 (2012).

[11] S. N. Liddick, A. Spyrou, B. P. Crider, F. Naqvi, A. C. Larsen, M. Guttormsen, M. Mumpower, R. Surman, G. Perdikakis, D. L. Bleuel et al., Phys. Rev. Lett. 116, 242502 (2016).

[12] M. Salvatores, G. Aliberti, M. Dunn, A. Hogenbirk, A. Ignatyuk, I. Ishikawa, I. Kodeli, A. J. Koning, R. McKnight,
R. W. Mills et al., Technical Report, OECD-NEA (2008), https: //www.oecd-nea.org/science/wpec/volume26/volume26.pdf.

[13] G. Aliberti, G. Palmiotti, M. Salvatores, T. K. Kim, T. A. Taiwo, M. Anitescu, I. Kodeli, E. Sartori, J. C. Bosq, and J. Tommasi, Ann. Nucl. Energy 33, 700 (2006).

[14] The Committee on the Assessment of and Outlook for Nuclear Physics; Board on Physics and Astronomy, Division on Engineering and Physical Sciences, National Research Council, Nuclear Physics: Exploring the Heart of Matter (The National Academies Press, Washington, DC, 2013).

[15] A. Couture and R. Reifarth, At. Data Nucl. Data Tables 93, 807 (2007).

[16] H. Beer and F. Käppeler, Phys. Rev. C 21, 534 (1980).

[17] E. Uberseder, R. Reifarth, D. Schumann, I. Dillmann, C. D. Pardo, J. Görres, M. Heil, F. Käppeler, J. Marganiec, J. Neuhausen et al., Phys. Rev. Lett. 102, 151101 (2009).

[18] M. Heil, F. Käppeler, E. Uberseder, R. Gallino, and M. Pignatari, Phys. Rev. C 77, 015808 (2008).

[19] R. Reifarth and Y. A. Litvinov, Phys. Rev. ST Accel. Beams 17, 014701 (2014).

[20] W. Hauser and H. Feshbach, Phys. Rev. 87, 366 (1952).

[21] T. Rauscher and F.-K. Thielemann, At. Data Nucl. Data Tables 79, 47 (2001).

[22] A. Couture, R. F. Casten, and R. B. Cakirli, Phys. Rev. C 96, 061601(R) (2017). 
[23] I. Dillmann, R. Plag, F. Käppeler, and T. Rauscher, in EFNUDAT Fast Neutrons - Proceedings of the Scientific Workshop on Neutron Measurements, Theory \& Applications, edited by F.-J. Hambsch (European Union, Luxembourg, 2009), http://www.kadonis.org.

[24] M. Wang, G. Audi, F. Kondev, W. Huang, S. Naimi, and X. Xu, Chin. Phys. C 41, 030003 (2017).
[25] W. J. Huang, G. Audi, M. Wang, F. G. Kondev, S. Naimi, and X. Xu, Chin. Phys. C 41, 030002 (2017).

[26] M. Chadwick, M. Herman, P. Obložinský, M. Dunn, Y. Danon, A. Kahler, D. Smith, B. Pritychenko, G. Arbanas, R. Arcilla et al., Nucl. Data Sheets 112, 2887 (2011).

[27] B. Pritychenko, Maxwellian-averaged cross sections and astrophysical reaction rates (2019), https://www.nndc.bnl.gov/astro/. 